MARCH 1989, PAGES 1-8

\title{
AN EXISTENCE AND UNIQUENESS THEOREM IN QUASI-STATIC VISCOELASTICITY*
}

\author{
BY \\ MAURO FABRIZIO \\ Dipartimento di Matematica, Piazza Porta S. Donato, Bologna, Italy
}

In some recent works, G. Fichera $[1,2]$ has raised the question of whether unidimensional quasi-static problems are well posed for linearly viscoelastic materials. These problems have the following natural generalization to the three-dimensional case:

$$
\begin{gathered}
\nabla \cdot \mathbf{T}(x, t)+\mathbf{f}(x, t)=0 \quad \text { on } Q=\Omega \times(-\infty, \infty), \\
\mathbf{T}(x, t)=\mathbf{G}(x, 0) \mathbf{E}(x, t)+\int_{0}^{\infty} \mathbf{G}^{\prime}(x, s) \mathbf{E}(x, t-s) d s, \\
\mathbf{u}(x, t)=0 \quad \text { on } \partial \Omega \times(-\infty, \infty) .
\end{gathered}
$$

Here, $\Omega$ is a regular open connected subset of the Euclidean space $\mathbf{R}^{3}$, $\mathbf{u}$ is the displacement vector, $\mathbf{E}=\frac{1}{2}\left(\nabla \mathbf{u}+\nabla \mathbf{u}^{\mathrm{T}}\right)$, $\mathbf{T}$ is the Cauchy stress-tensor, $\mathbf{f}$ is the body force, and for each $x \in \Omega, \mathbf{G}(x, \cdot)$ is a specified material function called the relaxation function; $\mathbf{G}^{\prime}$ is the derivative of $\mathbf{G}$ with respect to the elapsed time $s$, i.e.,

$$
\mathbf{G}(x, s)=\mathbf{G}(x, 0)+\int_{0}^{s} \mathbf{G}^{\prime}(x, \tau) d \tau .
$$

Fichera's examples are compatible with the assumption that $\mathbf{G}(x, 0)$ is symmetric and positive definite and that the relaxation function $\mathbf{G}(x, s)$ satisfies the hypotheses of the theory of Fading Memory formulated in $[3,4,5]$.

In two recent works A. Morro and I [6,7], pointed out that examples for $\mathbf{G}(x, \cdot)$, considered by Fichera in [2] and by us in [7], are not compatible with a certain form of the Second Law of Thermodynamics that permits equality only for reversible processes. Indeed, this principle places strong restrictions on the choice of $\mathbf{G}$.

In this paper I show that if the material is a solid, i.e., $\mathbf{G}(x, \infty)>\mathbf{0}$, and if the function $\mathbf{G}$ is compatible with the Second Law of Thermodynamics, i.e., $\int_{0}^{\infty} \mathbf{G}^{\prime}(s) \sin \omega s d s$ $>\mathbf{0}$ for all $\omega>0$, the problem (1), (2), (3) is well posed, namely just these two hypotheses induce a theorem of uniqueness and existence for this problem.

The study of the analytical problem therefore shows that in order to avoid the difficulties pointed out in the works of Fichera it is necessary to impose the restriction of the Second Law of Thermodynamics and, moreover, to formulate this principle in order to distinguish reversible from irreversible processes and to permit equality in the Second Law if and only if the processes are reversible. 
Quasi-static problem. Uniqueness and existence. A continuous viscoelastic body $\mathscr{B}$ is identified with a bounded and "regular" domain $\Omega$ of the tridimensional Euclidean space $\mathbf{R}^{3}$. Moreover, we consider unidimensional diplacements $u: Q \rightarrow R$, with $Q=\Omega \times(-\infty, \infty)$; therefore, the motion equations for the quasi-static problem, with Dirichlet conditions, will be:

$$
\begin{aligned}
& \nabla \cdot(\mathbf{G}(x, 0) \nabla u(x, t))+\nabla \cdot \int_{0}^{\infty} \mathbf{G}^{\prime}(x, s) \nabla u(x, t-s) d s=f(x, s), \\
& \left.u\right|_{\partial \Omega}=0 .
\end{aligned}
$$

Definition. A function $u$ is called a strict solution of the quasi-static problem (4) with Dirichlet boundary conditions (5) and source function $f$ in $L^{1}\left(-\infty, \infty ; L^{2}(\Omega)\right.$ ), if $u$ belongs to $L^{1}\left(-\infty, \infty ; H_{0}^{1}(\Omega)\right)$ and satisfies (4) and (5) almost everywhere in $Q=\Omega \times(-\infty, \infty)$.

The assumptions on $\mathbf{G}$ are:

(i) $\mathbf{G}(\cdot, \cdot)$ is continuous on $\bar{\Omega} \times[0, \infty)$, and differentiable in $\Omega \times(0, \infty)$.

(ii) $\mathbf{G}(x, 0)$ and $\mathbf{G}(x, \infty)=\mathbf{G}(x, 0)+\int_{0}^{\infty} \mathbf{G}^{\prime}(x, s)$ are symmetric tensors and are positive, i.e., there exist two positive constants $\delta_{1}, \delta_{2}$ such that at each $x$ :

$$
\mathbf{a} \cdot \mathbf{G}(x, 0) \mathbf{a} \geq \delta_{1} \mathbf{a} \cdot \mathbf{a}, \quad \mathbf{a} \cdot \mathbf{G}(x, \infty) \mathbf{a} \geq \delta_{2} \mathbf{a} \cdot \mathbf{a},{ }^{1}
$$

(iii) Graffi's inequality, i.e.:

$$
\mathbf{a} \cdot\left(\int_{0}^{\infty} \mathbf{G}^{\prime}(x, s) \sin \omega s d s\right) \mathbf{a}<0 \text { for all } \mathbf{a} \in V^{3} \backslash\{\mathbf{0}\} \text { and } \omega>0,
$$

where, for simplicity of exposition, it will be here assumed that the tensor $\mathbf{G}^{\prime}(x, s)$ is symmetric at each $x$ for all $s$. The present results can be proved provided only that $\mathbf{G}(x, 0)$ and $\mathbf{G}(x, \infty)$ are symmetric.

To explain the significance of assumption (iii) let me consider, for a moment, the one-dimensional case of a spatially homogeneous material, for which (7) would take the form:

$$
\int_{0}^{\infty} G^{\prime}(s) \sin \omega s d s<0, \quad \text { for all } \omega>0 .
$$

It is known that the relation

$$
\int_{0}^{\infty} G^{\prime}(s) \sin \omega s d s \leq 0, \quad \text { for all } \omega>0,
$$

is a necessary and sufficient ${ }^{2}$ condition that the work in cyclic processes be nonnegative. ${ }^{3}$

THEOREM 1. (Uniqueness) If $u$ is a strict solution with $f=0$, then $u=0$ a.e. in $Q$.

Proof. If $f=0$, then $u$ is a solution to the problem

$$
\begin{aligned}
& \nabla \cdot(\mathbf{G}(x, 0) \nabla u(x, t))+\nabla \cdot \int_{0}^{\infty} \mathbf{G}^{\prime}(x, s) \nabla u(x, t-s) d s=0, \\
& \left.u\right|_{\partial \Omega}=0 .
\end{aligned}
$$

\footnotetext{
1 These conditions follow from energy arguments and from the definition of a viscoelastic solid [8]. However, the physical compatibility of these hypotheses will be developed in the next section.

${ }^{2}$ See Graffi [9], Fabrizio-Morro [6].

${ }^{3}$ The Second Law of Thermodynamics for isothermal processes requires that the work done in a cyclic process be nonnegative. See, e.g., [6].
} 
The Fourier transform of (9) is given by

$$
\begin{aligned}
& \nabla \cdot \mathbf{G}(x, 0) \nabla \hat{u}(x, \omega)+\nabla \cdot \hat{\mathbf{G}}^{\prime}(x, \omega) \nabla \hat{u}(x, \omega)=0, \quad \omega \in \mathbf{R}, \\
& \left.\hat{u}\right|_{\partial \Omega}=0,
\end{aligned}
$$

where

$$
\hat{u}(x, \omega)=\frac{1}{\sqrt{2 \pi}} \int_{0}^{\infty} u(x, t) e^{i \omega t} d t, \quad \hat{\mathbf{G}}^{\prime}(x, \omega)=\int_{0}^{\infty} \mathbf{G}^{\prime}(x, t) e^{i \omega t} d t .
$$

Equation (10) can be rewritten as

$$
\nabla \cdot\left[\mathbf{G}(x, 0)+\hat{\mathbf{G}}^{\prime}(x, \omega)\right] \nabla \hat{u}(x, \omega)=0, \quad \omega \in \mathbf{R} .
$$

This equation is elliptic (see [10], [11]) because the symmetric tensor $[\mathbf{G}(x, 0)+$ $\left.\hat{\mathbf{G}}^{\prime}(x, \omega)\right]$ is such that, for an arbitrary vector $\mathbf{a} \in \mathbf{R}^{3} \backslash\{0\}$, the condition

$$
\left(\mathbf{G}(x, 0)+\hat{\mathbf{G}}^{\prime}(x, \omega)\right) \mathbf{a} \cdot \mathbf{a} \neq 0, \quad \text { for all } x \in \Omega \text { and } \omega \in \mathbf{R},
$$

holds. From (iii), when $\omega \neq 0$, the imaginary part of (13) satisfies

$$
\operatorname{Im}\left(\mathbf{G}(x, 0)+\hat{\mathbf{G}}^{\prime}(x, \omega)\right) \mathbf{a} \cdot \mathbf{a}=\left(\int_{0}^{\infty} \mathbf{G}^{\prime}(x, s) \sin \omega s d s\right) \mathbf{a} \cdot \mathbf{a} \neq 0 .
$$

For $\omega=0$ we obtain by (13)

$$
\operatorname{Re}\left(\mathbf{G}(x, 0)+\hat{\mathbf{G}}^{\prime}(x, 0)\right) \mathbf{a} \cdot \mathbf{a}=\mathbf{G}(x, 0)+\int_{0}^{\infty}\left[\mathbf{G}^{\prime}(x, s) d s\right] \mathbf{a} \cdot \mathbf{a}=\mathbf{G}(x, \infty) \mathbf{a} \cdot \mathbf{a}>0
$$

because from condition (ii) the tensor $\mathbf{G}(x, \infty)$ is symmetric and positive definite.

Therefore the ellipticity condition (13) implies that the problem (10), (11) has a unique solution $\hat{u}(x, \omega)=0$ for any $\omega \in \mathbf{R}$. Then the solution $u(x, t)$ of the problem $(8),(9)$ is given by

$$
u(x, t)=\frac{1}{\sqrt{2 \pi}} \int_{-\infty}^{\infty} \hat{u}(x, \omega) e^{-i \omega t} d \omega .
$$

Necessarily we obtain $u(x, t)=0$ on $Q$. By linearity, this proves the uniqueness of the solution of the quasi-static problem (5), (6).

Theorem 2. (Existence) The quasi-static problem with Dirichlet boundary conditions (4), (5) has a strict solution for each $f \in L^{1}\left(-\infty, \infty, L^{2}(\Omega)\right)$.

Proof. Applying the Fourier transform to the system (5), (6) we have

$$
\begin{gathered}
\nabla \cdot\left(\mathbf{G}(x, 0)+\hat{\mathbf{G}}^{\prime}(x, \omega)\right) \nabla \hat{u}(x, \omega)=\hat{f}(x, \omega), \quad x \in \Omega, \quad \omega \in \mathbf{R}, \\
\left.\hat{u}(x, \omega)\right|_{\partial \Omega}=0,
\end{gathered}
$$

where

$$
\hat{f}(x, \omega)=\frac{1}{\sqrt{2 \pi}} \int_{-\infty}^{\infty} f(x, t) e^{i \omega t} d t
$$

The ellipticity of Eq. (17) is not sufficient for existence for the problem (17), (18). Now it is necessary to prove that the coefficients are uniformly elliptic, namely for any $\omega \in \mathbf{R}$ there exist two constants $\gamma_{1}, \gamma_{2} \in \mathbf{R}^{++}$independent of $\omega$, such that one of the following two inequalities is satisfied:

$$
\left(\mathbf{G}(x, 0)+\int_{0}^{\infty} \mathbf{G}^{\prime}(x, s) \cos \omega s d s\right) \mathbf{a} \cdot \mathbf{a} \geq \gamma_{1} \mathbf{a} \cdot \mathbf{a},
$$




$$
-\left(\omega \int_{0}^{\infty} \mathbf{G}^{\prime}(x, s) \sin \omega s d s\right) \mathbf{a} \cdot \mathbf{a} \geq \gamma_{2} \mathbf{a} \cdot \mathbf{a},
$$

for all $\mathbf{a} \in \mathbf{R}^{3}$.

It follows from the Riemann-Lebesgue lemma that the quantities

$$
\begin{aligned}
& \hat{\mathbf{G}}_{1}^{\prime}(x, \omega)=\int_{0}^{\infty} \mathbf{G}^{\prime}(x, s) \cos \omega s d s, \\
& \hat{\mathbf{G}}_{2}^{\prime}(x, \omega)=\int_{0}^{\infty} \mathbf{G}^{\prime}(x, s) \sin \omega s d s
\end{aligned}
$$

are continuous in $\omega$ and go to zero for $\omega \rightarrow \pm \infty$.

Since, for $\omega=0$ and $\omega= \pm \infty$, the tensor $\left[\mathbf{G}(x, 0)+\hat{\mathbf{G}}_{1}^{\prime}(x, \omega)\right]$ is such that

$$
\begin{aligned}
\mathbf{G}(x, 0)+\hat{\mathbf{G}}_{1}^{\prime}(x, 0) & =\mathbf{G}(x, \infty), \\
\mathbf{G}(x, 0)+\hat{\mathbf{G}}_{1}^{\prime}(x, \pm \infty) & =\mathbf{G}(x, 0),
\end{aligned}
$$

it follows from the continuity of $\hat{\mathbf{G}}_{1}^{\prime}(x, \omega)$ and from $(6)$ that the tensor $\operatorname{Re}[\mathbf{G}(x, 0)+$ $\hat{\mathbf{G}}^{\prime}(x, \omega)$ ] is positive definite in some neighborhood $I_{0}$ of $\omega=0$. Similarly, $\operatorname{Re}\left(\mathbf{G}(x, 0)+\hat{\mathbf{G}}^{\prime}(x, \omega)\right)$ is positive definite in neighborhoods $I_{\infty}, I_{-\infty}$ of $\omega= \pm \infty$, because $\mathbf{G}(x, 0)$ is a positive definite tensor.

Therefore, the inequality (19) holds for all $\omega \in I_{0} \cup I_{\infty} \cup I_{-\infty}$. Finally by (14) and from the continuity of (22), the inequality (20) must be valid on $\mathbf{R} \backslash\left(I_{0} \cup I_{\infty} \cup I_{-\infty}\right)$.

By well-known theorems on elliptic equations, when the conditions (19), (20) are satisfied, the problem (17), (18) has a solution $\hat{u}(x, \omega)$ such that for any $\omega \in \mathbf{R}$, $\hat{u}(\cdot, \omega) \in H_{0}^{1}(\Omega)$ (see [10], [11]).

Using Green's functions we are able to prove that the inverse Fourier transform of the function $\hat{u}(x, \omega)$ exists.

We denote by $H\left(x, x^{\prime}, \omega\right)$ the Green's function for the problem

$$
\begin{gathered}
\nabla \cdot\left(\mathbf{G}(x, 0)+\hat{\mathbf{G}}^{\prime}(x, \omega)\right) \nabla H\left(x, x^{\prime}, \omega\right)=\delta\left(x-x^{\prime}\right), \\
\left.H\left(x, x^{\prime}, \omega\right)\right|_{\partial \Omega}=0,
\end{gathered}
$$

from which

$$
\hat{u}(x, \omega)=\int_{\Omega^{\prime}} \hat{f}\left(x^{\prime}, \omega\right) H\left(x, x^{\prime}, \omega\right) d x^{\prime} .
$$

For any fixed $\omega \in \mathbf{R}$ the function $H\left(x, x^{\prime}, \omega\right)$ is an element of $H_{0}^{1}$ (see [11]). Moreover, $H\left(x, x^{\prime}, \omega\right)$ is continuous in $\omega$, because the coefficients $\hat{\mathbf{G}}^{\prime}(x, \omega)$ are continuous in $\omega$ on $\mathbf{R}$ [12]. Since $\hat{\mathbf{G}}^{\prime}(x, \omega) \rightarrow 0$ when $\omega \rightarrow \pm \infty$, then $\lim _{\omega \rightarrow \pm \infty} H\left(x, x^{\prime}, \omega\right)$ exists and belongs to $H_{0}^{1}$, because it is a solution of the following problem:

$$
\begin{gathered}
\nabla \cdot\left(\mathbf{G}(x, 0) \nabla H\left(x, x^{\prime}, \pm \infty\right)\right)=\delta\left(x-x^{\prime}\right), \\
\left.H\left(x, x^{\prime}, \pm \infty\right)\right|_{\partial \Omega}=0 .
\end{gathered}
$$

Hence $H\left(x, x^{\prime}, \omega\right)$ will be bounded in $\omega$.

By $(27) \hat{u} \in L^{1}\left(-\infty, \infty ; H_{0}^{1}(\Omega)\right)$ admits an inverse Fourier transform

$$
u(x, t)=\frac{1}{\sqrt{2 \pi}} \int_{-\infty}^{\infty} \hat{u}(x, \omega) e^{i \omega t} d \omega
$$

such that $u \in L^{1}\left(-\infty, \infty ; H_{0}^{1}(\Omega)\right)$ is a solution of the original problem (5), (6). 
Thermodynamic restrictions in linear viscoelasticity. In a work with Morro [6] we proved that the relation (9) represents a necessary and sufficient condition for the validity of the Second Law of Thermodynamics.

In a subsequent work [7] Morro and I have found an example of a $\mathbf{G}$ which satisfies (8), but such that there is an $\bar{\omega}>0$ with

$$
\int_{0}^{\infty} \mathbf{G}^{\prime}(x, s) \sin \bar{\omega} s d s=0 .
$$

For this particular G, in [7] it has been demonstrated that the problem (1)-(3) may have more than one solution in $L^{1}\left(-\infty, \infty ; H_{0}^{1}(\Omega)\right)$ and therefore the relative quasi-static problem is ill-posed.

This example leads us to consider the need of a formulation of the Second Law of Thermodynamics, that distinguishes reversible from irreversible processes.

The problem thus arises as to an appropriate definition of a reversible process and therefore to a more restrictive formulation of the Second Law in which equality holds only for reversible processes. Since it is natural to assume that in viscoelastic material only the static processes can be reversible, according to the Second Law of Thermodynamics, in the one-dimensional case it is possible to prove the validity of $\left(7^{\prime}\right)$ instead of (8).

Such a formulation permits us, therefore, to achieve a perfect harmony between the well posedness of the quasi-static problem and the thermodynamic restrictions on the relaxation function $\mathbf{G}$.

A material is said to be linearly viscoelastic if the constitutive relation is the following:

$$
\mathbf{T}(x, t)=\mathbf{G}(x, 0) \mathbf{E}(x, t)+\int_{0}^{\infty} \mathbf{G}^{\prime}(x, s) \mathbf{E}^{t}(s) d s,
$$

where $\mathbf{E}^{t}(s):=\mathbf{E}(t-s), s \in[0, \infty)$, is called history of the infinitesimal strain tensor.

The history space

$$
\Sigma=\left\{\mathbf{E}^{t}:[0, \infty) \rightarrow \operatorname{Sym}(V) ; \int_{0}^{\infty}\left|\mathbf{E}^{t}(s)\right|^{2} h(s) d s<\infty\right\}
$$

is a Banach space under the norm

$$
\left|\mathbf{E}^{t}\right|=|\mathbf{E}(t)|^{2}+\int_{0}^{\infty}\left|\mathbf{E}^{t}(s)\right|^{2} h(s) d s,
$$

where $h:[0, \infty) \rightarrow \mathbf{R}^{+}$is such that $h \in L^{1}(0, \infty)$, and $\lim _{s \rightarrow \infty} s^{2} h(s)=0$.

Definition. A function $P:\left[0, d_{P}\right) \rightarrow \operatorname{Sym}(V)$ which is piecewise continuous and is defined for any $t \in\left[0, d_{P}\right]$ as

$$
P(t):=\dot{\mathbf{E}}_{P}(t)
$$

is called a deformation process; the number $d_{P} \in \mathbf{R}^{+}$is called the duration of the process.

In the following we often use the process $P_{t}$ defined as the restriction of the process $P:\left[0, d_{P}\right) \rightarrow \operatorname{Sym}(V)$ to the interval $[0, t) \subset\left[0, d_{P}\right)$. Let $\Pi$ be the set of all accessible processes of the material while the state $\sigma$ for a viscoelastic material is given by the history $\mathbf{E}^{t}$, i.e.,

$$
\sigma=\mathbf{E}^{t}
$$


and therefore $\Sigma$ will be the state space.

Fixing a pair $(\sigma, P)$, we consider the path in the state space defined by means of all states obtained when, leaving from the initial state $\sigma$, we follow the process $P$. It is possible to define such a concept by means of the transformation function $\hat{\rho}: \Sigma \times \Pi \rightarrow \Sigma$ defined by

$$
\hat{\rho}(\sigma, P)=\left(\mathbf{E}\left(t+d_{P}\right), \mathbf{E}^{t+d_{P}}\right)
$$

where, if $\sigma=\left(\mathbf{E}(t), \mathbf{E}^{t}\right)$, then

$$
\begin{gathered}
\mathbf{E}\left(t+d_{P}\right)=\int_{t}^{t+d_{P}} \dot{\mathbf{E}}_{P}(t) d \tau+\mathbf{E}(t), \\
\mathbf{E}^{t+d_{P}}(s)= \begin{cases}\int_{t}^{t+d_{P}-s} \dot{\mathbf{E}}_{P}(r) d r+\mathbf{E}(t), & s \in\left[0, d_{P}\right) \\
\mathbf{E}^{t}\left(s-d_{P}\right) & s \in\left(d_{P}, \infty\right) .\end{cases}
\end{gathered}
$$

The curve $\mathscr{C}$ in the state space built by means of the pair $(\sigma, P)$ is given by

$$
\sigma_{t}=\hat{\rho}\left(\sigma, P_{t}\right), \quad t \in\left[0, d_{P}\right) .
$$

Definition. A pair $(\sigma, P) \in \Sigma \times \Pi$ is called a cyclic process if $\hat{\rho}(\sigma, P)=\sigma$.

We denote by $W$ the power of the stress

$$
W\left(\sigma_{t}, P(t)\right)=\mathbf{T}\left(\sigma_{t}\right) \cdot \dot{\mathbf{E}}(t)
$$

and by $L(\sigma, P)$ the work on the curve $\mathscr{C}$ performed by going from $\sigma$ to $\hat{\rho}(\sigma, P)$ via the process $P$, i.e.,

$$
L(\sigma, P)=\int_{\mathscr{C}} \mathbf{T}\left(\sigma_{t}\right) \cdot \dot{\mathbf{E}}(t) d t
$$

Definition. A cyclic process $(\sigma, P)$ is called reversible if the new process $P^{-}$ defined in $\left[0, d_{P}\right)$ as

$$
P^{-}(\tau)=-\dot{\mathbf{E}}\left(d_{P}-\tau\right), \quad \tau \in\left[0, d_{P}\right),
$$

belongs to $\Pi$ and, moreover,

a) $\hat{\rho}\left(\sigma, P_{t}\right)=\hat{\rho}\left(\sigma, P_{d_{P}-t}^{-}\right)$,

b) $w\left(\sigma_{t}, P(t)\right)=-w\left(\sigma_{t}, P_{d_{P}-t}^{-}\right)$.

REMARK. For viscoelastic materials the class of reversible processes contains only sequences of equilibrium states.

As shown in [6], it is sufficient to express the Second Law of Thermodynamics only on cyclic processes when the material is viscoelastic.

Second Law of Thermodynamics. On any cyclic process $(\sigma, P) \in \Sigma \times \Pi$ on the state space corresponding to the closed curve $\mathscr{C}$, we have

$$
\int_{\mathscr{C}} \mathbf{T}\left(\sigma_{t}\right) \cdot \dot{\mathbf{E}}(t) d t \geq 0,
$$

where equality holds if and only if the cyclic process is reversible.

To obtain the restrictions on the function $\mathbf{G}(s)$ as a consequence of this principle we consider periodic fields

$$
\tilde{\mathbf{E}}(t)=\mathbf{E}_{1} \cos \omega t+\mathbf{E}_{2} \sin \omega t, \quad \omega \neq 0
$$


It is possible to associate with (35) the state

$$
\tilde{\sigma}=\tilde{\mathbf{E}}^{t}(s)=\mathbf{E}_{1} \cos \omega(t-s)+\mathbf{E}_{2} \sin \omega(t-s)
$$

and the process $\tilde{P} \in \Pi$ of duration $d_{P}=2 \pi m / \omega$, with $m \in \mathbf{N}$, is defined as

$$
\tilde{P}(t)=-\omega \mathbf{E}_{1} \sin \omega t+\omega \mathbf{E}_{2} \cos \omega t .
$$

Of course a pair $(\tilde{\sigma}, \tilde{P})$ constitutes a cyclic process if

$$
\hat{\rho}(\tilde{\sigma}, \tilde{P})=\tilde{\sigma} .
$$

REMARK. Such cyclic processes always are irreversible for every $\omega \neq 0$.

Following the same argument used in [6], it is possible to determine the restrictions that the inequality (34) imposes on the function $\mathbf{G}$ (see [6]).

THEOREM 3. A necessary condition for the validity of the Second Law is that the inequality

$$
\begin{aligned}
\mathbf{E}_{1} \cdot\left(\mathbf{G}^{\mathrm{T}}(0)-\mathbf{G}(0)\right) \mathbf{E}_{2} & -\int_{0}^{\infty}\left(\mathbf{E}_{1} \cdot \mathbf{G}^{\prime}(s) \mathbf{E}_{1}+\mathbf{E}_{2} \mathbf{G}^{\prime}(s) \mathbf{E}_{2}\right) \sin \omega s d s \\
& -\int_{0}^{\infty} \mathbf{E}_{1} \cdot\left(\mathbf{G}^{\prime}(s)-\mathbf{G}^{\mathrm{T}}(s)\right) \mathbf{E}_{2} \cos \omega s d s>0
\end{aligned}
$$

holds for every $\omega \in \mathbf{R}^{++}$and every $\mathbf{E}_{1}, \mathbf{E}_{2} \in \operatorname{Sym}(V)$.

Corollary. If in (37) we let $\omega \rightarrow \infty$ and then $\omega \rightarrow 0$ we shall have, respectively,

$$
\begin{aligned}
\mathbf{G}(0) & =\mathbf{G}^{\mathrm{T}}(0), \\
\mathbf{G}(\infty) & =\mathbf{G}^{\mathrm{T}}(\infty) .
\end{aligned}
$$

REMARK. It is also possible to prove, still following thermodynamic considerations, that (see [6])

$$
[\mathbf{G}(0)-\mathbf{G}(\infty)] \mathbf{a} \cdot \mathbf{a} \geq 0 \quad \text { for all } \mathbf{a} \in \operatorname{Sym}(V) .
$$

Finally we shall assume the time-reversal condition formulated by Day [13] is satisfied. This implies that

$$
\mathbf{G}^{\prime}(s)=\mathbf{G}^{\prime T}(s) \quad \text { for all } \quad s \in(0, \infty) .
$$

Corollary. Using the properties of symmetry (38) and (41) it follows from (37) that the Second Law can be formulated as follows:

$$
\mathbf{E} \cdot \int_{0}^{\infty} \mathbf{G}^{\prime}(s) \sin \omega s d s \mathbf{E}>0 \quad \text { for all } \quad \mathbf{E} \in \operatorname{Sym}(v), \quad \omega \in \mathbf{R}^{++} .
$$

Now considering a static deformation state, namely a history for which

$$
\mathbf{E}^{t}(s)=\mathbf{E}_{0} \neq 0, \quad s \in[0, \infty),
$$

the corresponding stress is given by

$$
\mathbf{T}(\infty)=\left(\mathbf{G}(0)+\int_{0}^{\infty} \mathbf{G}^{\prime}(s) d s\right) \mathbf{E}_{0}=\mathbf{G}(\infty) \mathbf{E}_{0} .
$$

In this way we find for static processes a constitutive equation of elastic type. 
REMARK. Since the viscoelastic material is supposed to be solid, corresponding to the static deformation $\mathbf{E}_{0}$ we have a nonzero value of the stress tensor $\mathbf{T}$. This implies that

$$
\mathbf{G}(\infty) \neq \mathbf{0} \text {. }
$$

Moreover, the scalar product $\mathbf{T}(\infty) \cdot \mathbf{E}_{0}$ will be strictly positive.

Therefore, as in the elastic case, we have

$$
\mathbf{T}(\infty) \cdot \mathbf{E}_{0}=\mathbf{G}(\infty) \mathbf{E}_{0} \cdot \mathbf{E}_{0}>0 .
$$

RemarK. It follows from (45) that $\mathbf{G}(\infty)$ is not only symmetric, but also positive definite. From this we are able to prove by means of (40) that also $\mathbf{G}(0)$ must be positive definite.

Acknowledgment. This work has been partially supported by Italian M.P.I. 40\% project and G.N.F.M. of C.N.R.

\section{REFERENCES}

[1] G. Fichera, Avere una memoria tenace crea gravi problemi, Arch. Rat. Mech. Anal. 70, 101-112 (1979)

[2] G. Fichera, Sul Principio della memoria evanescente, Rend. Sem. Mat. Univ. Padova 68, 245-259 (1982)

[3] B. D. Coleman and W. Noll, Foundations of linear viscoelasticity, Rev. Modern Phys. 33, 239-249 (1961)

[4] B. D. Coleman, On thermodynamics, strain impulses, and viscoelasticity, Arch. Rat. Mech. Anal. 17, 230-254 (1964)

[5] B. D. Coleman and V. J. Mizel, Norms and semi-groups in the theory of fading memory, Arch. Rat. Mech. Anal. 23, 87-123 (1966)

[6] M. Fabrizio and A. Morro, Viscoelastic relaxation functions compatible with thermodynamics, J. Elasticity 19, 63-75 (1988)

[7] A. Morro and M. Fabrizio, On uniqueness in linear viscoelasticity: A family of counterexamples, Quart. Appl. Math. 45, 321-325 (1987)

[8] A. C. Pipkin, Lectures on viscoelasticity theory, Springer, Berlin, 1972

[9] D. Graffi, Sui problemi della ereditarietà lineare, Nuovo Cimento 5, 53-71 (1928)

[10] G. Fichera, Existence theorems in elasticity, Handbuch der Physik, vol. VI a/2, Springer, Heidelberg, 1972, pp. 347-389

[11] F. Treves, Basic linear partial differential equations, Academic Press, New York, 1975

[12] M. S. Agranovich and M. I. Vishik, Elliptic boundary-value problems depending on a parameter, Dokl. Akad. Nauk SSSR 149, 223-226 (1963)

[13] W. A. Day, The thermodynamics of simple materials with fading memory, Springer Tracts in Natural Philosophy 22, Springer-Verlag, New York-Heidelberg, 1972 